\title{
Frataxin point mutations in two patients with Friedreich's ataxia and unusual clinical features
}

\author{
Melissa L McCormack, Rodney P Guttmann, Matthew Schumann, Jennifer M Farmer, \\ Catherine A Stolle, Victoria Campuzano, Michel Koenig, David R Lynch
}

\author{
Departments of \\ Neurology, Pediatrics, \\ and Pharmacology, \\ Division of \\ Neuroscience \\ Research, Children's \\ Hospital of \\ Philadelphia, \\ Pennsylvania , USA \\ M L McCormack \\ R P Guttmann \\ M Schumann \\ D R Lynch \\ Division of Medical \\ Genetics, University of \\ Pennsylvania School of \\ Medicine, \\ Philadelphia, \\ Pennsylvania 19104, \\ USA \\ J Farmer
}

Department of

Genetics

C Stolle

Institut de Genetique

et de Biologie

Moleculaire et

Cellulaire (IGBMC),

CNRS, INSERM,

Universite Louis

Pasteur, Strasbourg,

France

V Campuzano

M Koenig

Correspondence to: Dr David R Lynch, Division

of Neuroscience Research,

Children's Hospital of

Philadelphia, 502 Abramson

Building, Philadelphia,

Pennsylvania 19104-4318,

USA

lynch@pharm.med.upenn.edu

Received 17 May 1999 and in final form

3 December 1999

Accepted 14 December 1999

\begin{abstract}
Two patients with a progressive ataxia are presented with clinical features consistent with classic Friedreich's ataxia (FRDA), but also with features unusual for FRDA. Analysis of DNA showed that each patient is heterozygous for the expanded GAA repeat of FRDA, but carries a base change on his other frataxin allele. For one patient a non-conservative arginine to cysteine amino acid change is predicted at amino acid 165 whereas the other mutation is found at the junction of exon one and intron one. Muscle biopsy showed an absence of frataxin immunoreactivity in the patient harbouring the intronic mutation, confirming the pathological nature of the base change. These mutations extend the range of point mutations seen in FRDA, and agree with recent reports suggesting phenotypic variation in patients with FRDA harbouring point mutations in conjunction with an expanded GAA repeat.

(F Neurol Neurosurg Psychiatry 2000;68:661-664)
\end{abstract}

Keywords: diabetes; optic neuropathy; neurogenetics

Friedreich's ataxia (FRDA) is an autosomal recessive ataxia, characterised by onset before the age of 25, progressive ataxia, absent tendon reflexes, dysarthria, extensor plantar responses, and distal weakness of the legs. ${ }^{1}$ Associated features include optic atrophy, sensorineural deafness, diabetes mellitus, pes cavus, scoliosis, and hypertrophic cardiomyopathy. Until recently, diagnosis depended on clinical criteria and was complicated by phenotypic variability. Campuzano et al identified the most common mutation causing FRDA as an expanded GAA repeat in the gene for the protein frataxin. Ninety six per cent of patients are homozygous for the GAA expansion. Frataxin point mutations (missense, nonsense, and intronic) are found in patients who are heterozygous for the expanded allele. ${ }^{2-6}$ Whereas the expanded repeat may produce a loss of function phenotype by altering RNA transcription, the point mutations also result in a loss of function phenotype as no frataxin is found on western blots of muscle tissue from patients carrying these mutations. ${ }^{4}$ The phenotypic features of patients harbouring point mutations may differ slightly from classic FRDA. ${ }^{7}$ We present two patients with phenotypes similar to FRDA but with differences which caused clinical confusion. On molecular testing each patient was shown to be heterozygous for the GAA expansion of FRDA with a point mutation of his second FRDA allele.

\section{Methods}

GENOMIC DNA ISOLATION

GAA repeat analysis was performed by the Genetics Diagnostic Laboratory at the University of Pennsylvania. ${ }^{2}$ Exons $1-5 a$ were amplified using the intronic primers described previously and sequenced using Sequenase ${ }^{\mathrm{TM}}$ polymerase chain reaction (PCR) product sequencing kit and automated sequencing. ${ }^{2}$

Western blots of quadriceps biopsy tissue were performed as described previously. ${ }^{4}$

\section{Results}

CASE HISTORIES

Patient 1

An 11 year old boy presented at the age of 5 with difficulty walking. This slowly progressed without complaints referable to his upper limbs. His lower limbs became stiff, and his feet progressively turned down and in. Speech, vision, and sensation were unchanged. No autonomic or cardiac symptoms were present. Three cousins of his maternal grandmother had a neurodegenerative disease similar to Friedreich's ataxia by report.

Physical examination disclosed a well developed boy without significant scoliosis but with bilateral pes cavus. Mental status and speech were normal. Optic disks were flat without temporal pallor. Visual acuity was $20 / 20$, and visual fields were full. Extraocular movements were full without nystagmus. No muscle atrophy was present. Tone was normal in the upper limbs, and increased in his lower limbs. The increased tone was uniform with passive movement and was not velocity dependent. Power was normal, although his feet were plantar flexed and inverted from increased tone. Reflexes were symmetrically increased at his knees, but absent in his upper limbs and at his ankles. Plantar responses were extensor bilaterally. Finger to nose testing was well 


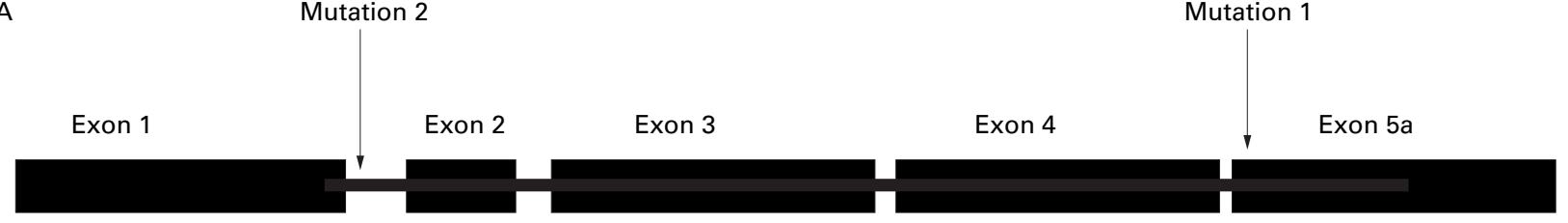

B

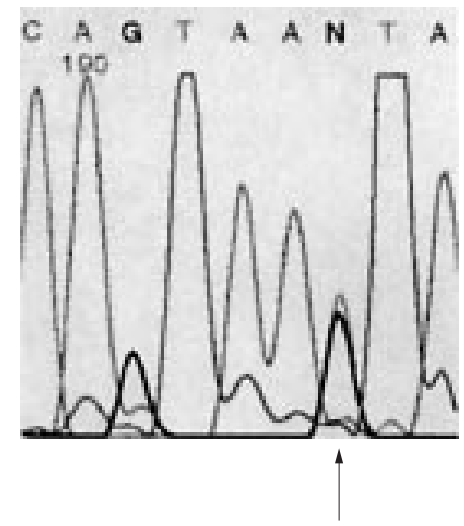

Patient 2

(G/C transversion)

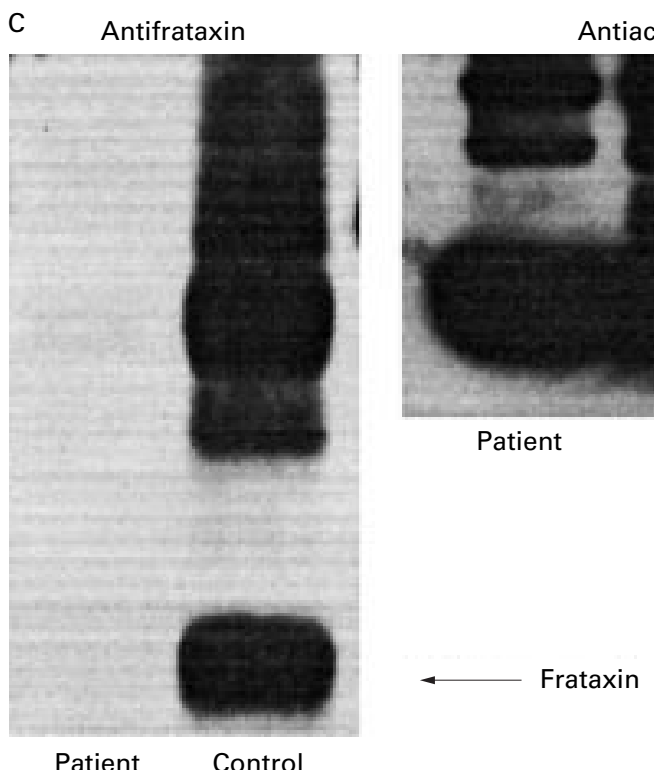

Location and confirmation of Frataxin point mutations. (A) The location of the mutations between exon 1 and exon 2 (patient 2) and in exon 5a (patient 1). (B) Direct DNA sequencing disclosed a $G$ to $C$ base change at the fifth base of intron 1 in patient 2 which was not found in controls. The same base change was seen in the asymptomatic brother of the patient. The brother has no expanded GAA repeat, unlike the patient, thus showing that these two mutations are on independent alleles. (C) Frozen muscle tissue from a quadriceps biopsy was analysed for frataxin immunoreactivity by western blotting. The patient's biopsy specifically lacked an $18 \mathrm{kD}$ band consistent with frataxin which was present in a patient without Friedreich's ataxia, who underwent biopsy for a myopathy. By contrast, levels of actin were immunologically identical in the two samples. Several higher molecular weight bands were present in the non-FRDA sample, consistent with previously noted alternative forms of frataxin or with postmortem aggregation.

performed; no pseudoathetosis was seen. He had decreased proprioception in the feet. The boy walked on his toes with stiff legs. His Romberg's sign was positive.

The patient was initially diagnosed with idiopathic dystonia. Electromyography and nerve conduction velocity were normal at age 7. A slight decrease in ulnar and median sensory potentials had developed by the age of 9 , and sural sensory potentials were absent at that time. An ECG showed inverted T waves in the inferior and left precordial leads. An echocardiogram demonstrated a slight increase in the left ventricular end diastolic dimension for age. A DNA test for FRDA showed a single expanded allele (1000 repeats), suggestive of an apparent carrier status. His mother was shown to be a carrier of an expanded allele, whereas his father had no expanded alleles. Sequencing of the patient's frataxin gene disclosed a single base change $(\mathrm{C} \rightarrow \mathrm{T})$ at base 493 , predicting an arginine to cysteine change at amino acid 165 in exon 5 a (figure A). No other base changes were present in the frataxin coding region. This base change was also present in his father, showing that the point mutation and the expanded GAA repeat were inherited as distinct alleles. We analysed this region of frataxin in 37 other people and found the $\mathrm{R} 165 \mathrm{C}$ change in none of them. This included six control subjects, six ataxic patients with no expanded alleles for frataxin, and 25 other patients with only one expansion of frataxin. Fourteen of the patients with one expanded allele had other mutations in the frataxin gene.

\section{Patient 2}

This 47 year old man was evaluated for an atypical progressive neurodegenerative disorder consisting of ataxia, sensorimotor axonal polyneuropathy, proprioceptive loss, optic neuropathy, and diabetes. Difficulty with writing and walking was noted around the age of 8 , and he developed progressive weakness in his lower limbs. He was diagnosed with Friedreich's ataxia, but this was later changed to CharcotMarie-Tooth disease because of increasing calf atrophy and a failure to develop speech dysfunction. Progression of his weakness led to confinement to a wheelchair at the age of 21 . Progressive visual difficulties were noted in early adulthood, including night blindness and diminished peripheral vision, especially in his lower visual fields. Diabetes was diagnosed at the age of 40 , with subsequent need for oral hypoglycaemic agents. There was no family history of neurological disease or consanguinity. No scoliosis or cardiomyopathy was present.

Neurological examination at the age of 40 disclosed a visual acuity of 20/30 OD and 20/40 OS with central scotomas OU and 
peripheral constriction of visual fields, the inferior greater than than the superior. Zero of 10 Ishihara colour plates were identified with each eye. Optic nerves were pale bilaterally, but no pigmentary retinal changes were present. The lens and anterior chambers were normal. Speech and language function were normal. He had marked atrophy of his lower limbs, distal greater than proximal, with corresponding weakness. Vibration sense was absent to the hips bilaterally and moderately reduced in the arms in a length dependent fashion. Proprioception was severely decreased in the lower limbs to the knee. Sensitivity to cold was decreased bilaterally in all his limbs. On extension of the arms pseudoathetosis was present, and dysmetria was present on finger to nose testing. Deep tendon reflexes were absent throughout, and plantar responses were absent bilaterally.

Electroretinography was normal. Motor nerve conduction velocities were normal. Sensory potentials were absent bilaterally in the radial, median, and ulnar nerves. Electromyography studies disclosed evidence of length dependent chronic denervation, consistent with a sensorimotor polyneuropathy with a greater sensory component or the combination of a sensory neuronopathy with a superimposed axonal neuropathy. A muscle biopsy showed type II fibre predominance and fibre type grouping, consistent with neurogenic atrophy. A serum $\alpha$-tocopherol concentration was $14.3 \mathrm{mg} / 1$ (normal). An ECG and echocardiogram were normal. Triplet repeat testing for Friedreich's ataxia was performed, disclosing an expansion of 670 repeats on one allele with the other allele of normal size.

DNA sequencing of the frataxin gene found a single base substitution ( $\mathrm{G} \rightarrow \mathrm{C}$ transversion) at the fifth base of intron 1 on one allele (figure A and B). No other abnormalities were found in sequencing exons $1-5$ and the adjacent portions of intervening introns. ${ }^{2}$ To confirm that this base change was on a distinct allele from the expanded repeat, we sequenced the frataxin gene in his brother, who has no neurological, cardiac, or endocrinological abnormalities. This man had the same base change, but lacked an expanded triplet repeat, suggesting that these mutations are inherited as distinct alleles. However, as this base change is found in an intronic portion of the frataxin gene, it could theoretically represent a silent polymorphism. We assessed this possibility by determining if the base change was found in a significant number of control subjects. Direct sequencing of the frataxin gene from 11 controls found this base change in none. We also assessed amplified exon 1 with the adjacent intronic region for the presence of Mae III sites as this base transversion adds one site. Frataxin exon 1 from 24 controls was cleaved by this enzyme into segments of 154 and 47 bases indicating the absence of an extra Mae III site in all controls. This suggests that this abnormality is not often found within the population.

Finally, to ensure that the base change found causes a pathological abnormality, we confirmed that this transversion produces a change in frataxin expression. No immunologically detectable frataxin was found in muscle using a monoclonal antibody against frataxin, whereas frataxin was found in muscle from a subject without FRDA (figure C). Muscle contained similar levels of actin in the patient and a control (figure C).

\section{Discussion}

In the present patients we have found two mutations which, in combination with an expansion of the naturally occurring GAA repeat within the frataxin gene on the other allele, are associated with the production of FRDA. We have shown that both base changes are on different alleles from the GAA expansion, consistent with their being causative mutations, although we cannot rule out the possibility that they are uncommon polymorphisms. In patient 1 , the base change predicts a non-conservative amino acid substitution, similar to other missense mutations reported in patients with FRDA. $^{7}$ Arginine 165 is also present in mouse and yeast frataxin, showing its high level of evolutionary conservation and arguing that it is a necessary amino acid. ${ }^{48}$ In the other patient, the intronic base change is associated with a loss of frataxin on muscle biopsy. This base change lies at a similar position to a previously reported intronic base change in frataxin between exons 4 and 5, and conceivably could disrupt RNA splicing or transcription.?

The use of genetic testing has increased the range of phenotypic features associated with FRDA. These features include later age of onset, spasticity rather than dorsal column sensory loss as a major manifestation, pure sensory ataxia, and generalised chorea. ${ }^{9-12}$ One recent report shows that unusual phenotypic features of FRDA are more common in patients having point mutations rather than two expanded triplet repeats. ${ }^{7}$ These unusual features include lack of dysarthria $(40 \%$ of compound heterozygous patients), retained reflexes (28\%), lack of bilateral extensor plantar responses $(24 \%)$, and optic nerve pallor $(28 \%){ }^{7}$ Missense mutations in the amino terminal portion of frataxin have been specifically associated with milder phenotypic features. ${ }^{7}$ The phenotypic features of the two patients presented here agree with these results. Our first patient differs from many patients with FRDA in the increased tone in his lower limbs, which directed evaluation away from FRDA. This patient is early in his course, and may develop more typical features later. Our second patient differs from many patients with FRDA in his lack of dysarthria, the significant motor neuropathy, and the significant optic atrophy, all of which caused difficulty in making a definitive diagnosis before the use of molecular approaches. Optic nerve abnormalities are seen on visual evoked responses in up to $64 \%$ of patients with FRDA, but only $5 \%-15 \%$ of patients have optic nerve dysfunction which is clinically significant. ${ }^{13}$ Thus, the unusual phenotypic features in our two patients agree with recent genotypephenotype correlations. 


\section{Note added in proof}

While this work was in press we became aware of one reported patient with FRDA with the R16SC mutation and two sisters with the mutation R16SP. ${ }^{14}{ }^{15}$ Consistent with the present work all three have atypical phenotypes.

This work was supported by Grant NIDA DAO7130, Fellowship 1F32-DAO5675, NS01789-01 from the Nationa Institutes of Health, a Junior Investigator Award from NARSAD, and a starter grant from MDA. We thank Drs Kenneth Fischbeck and Robert Wilson for critical reading of the manuscript.

1 Harding AE. Friedreich's ataxia: a clinical and genetic study of 90 families with an analysis of early diagnostic criteria and intrafamilial clustering of clinical features. Brain 1981;104:589-620.

2 Campuzano V, Montermini L, Molto MD, et al. Friedreich's ataxia: autosomal recessive disease caused by an intronic GAA triplet repeat expansion. Science 1996;271:1423-7.

3 Bidichandani SI, Ashizawa T, Patel PI. Atypical Friedreich ataxia caused by compound heterozygosity for a novel missense mutation and the GAA triplet-repeat expansion. Am f Hum Genet 1997;60:1251-6.

4 Campuzano V, Montermini L, Lutz Y, et al. Frataxin is reduced in Friedreich ataxia patients and is associated with reduced in Friedreich ataxia patients and is associated with 80 .

5 Bidichandani SI, Ashizawa T, Patel PI. The GAA tripletrepeat expansion in Friedreich ataxia interferes with transcription and may be associated with an unusual DNA structure. Am F Hum Genet 1998; 62:111-21.
6 Bartolo C, Mendell JR, Prior TW. Identification of a missense mutation in a Friedreich's ataxia patient: implications for diagnosis and carrier studies. Am $7 \mathrm{Med}$ Genet 1998; 79:396-399.

7 Cossee M, Durr A, Schmitt M, et al. Friedreich's ataxia: point mutations and clinical presentation of compound heterozygotes. Ann Neurol 1999;45:200-6.

8 Wilson RB, Roof DM. Respiratory deficiency due to loss of mitochondrial DNA in yeast lacking the frataxin homologue. Nat Genet 1997;17:215-17.

9 Berciano J, Combarros O, De Castro M, et al. Intronic GAA triplet repeat expansion in Friedreich's ataxia presenting with pure sensory ataxia. F Neurol 1997;244:390-1.

10 Ragno M, De Michele G, Cavalcanti F, et al. Broadened Friedreich's ataxia phenotype after gene cloning. Minimal GAA expansion causes late-onset spastic ataxia. Neurology 1997;49:1617-20.

11 Hanna MG, Davis MB, Sweeney MG, et al. Generalized chorea in two patients harboring the Friedreich's ataxia gene trinucleotide repeat expansion. Mov Disord 1998;13: $339-40$.

12 Montermini L, Richter A, Morgan K, et al. Phenotypic variability in Friedreich ataxia: role of the associated GAA triplet repeat expansion. Ann Neurol 1997;41:675-82.

13 Carroll WM, Kriss A, Baraitser M, et al. The incidence and nature of visual pathway involvement in Friedreich's ataxia. A clinical and visual evoked potential study of 22 patients. Brain 1980;103:413-34.

14 Forrest SM, Knight M, Delatycki MB, et al. The correlation of clinical phenotype in Friedreich ataxia with the site of point mutation in the FRDA gene. Neurogenetics 1998;1: 253-7.

15 De Michele G, Filla A, Cavalcanti F, et al. Atypical Friedreich ataxia phenotype associated with a novel missense mutation on the X25 gene. Neurology 2000;54:496-9. 